\title{
Preoptic Glutamate Facilitates Male Sexual Behavior
}

\author{
Juan M. Dominguez, Mario Gil, and Elaine M. Hull \\ Department of Psychology and Neuroscience Program, Florida State University, Tallahassee, Florida 32306-1270
}

The medial preoptic area (MPOA) is a critical regulatory site for the control of male sexual behavior. We first measured glutamate in 2 min microdialysate samples from the MPOA before, during, and after copulation by male rats. There was a slight $[\sim 140 \%$ of baseline $(\mathrm{BL})]$ rise in extracellular glutamate when the female was presented, a significant increase $(\sim 170 \%$ of $\mathrm{BL})$ during periods of mounting and intromitting, and a very large increase in samples collected during ejaculation ( $\sim 300 \%$ of BL$)$. A precipitous fall in levels occurred in the first postejaculatory sample; the magnitude of this fall was highly correlated with the length of the postejaculatory interval of quiescence. In experiment 2, we reverse-dialyzed a mixture of glutamate uptake inhibitors into the MPOA before and during mating; control animals received artificial CSF. The mixture increased extracellular glutamate ( $\sim 280 \%$ of $B L)$, increased the number of ejaculations in the 40 min test, decreased ejaculation latency, and decreased the postejaculatory latency to resume copulation. These data, together with other findings that glutamate in the MPOA can elicit genital reflexes in anesthetized rats and that glutamate receptor antagonists in the MPOA impair copulation, strongly suggest that MPOA glutamate is a major facilitator of copulation and that the postejaculatory fall in glutamate regulates the postejaculatory interval.

Key words: medial preoptic area; glutamate; sexual behavior; limbic system; male rats; HPLC

\section{Introduction}

The medial preoptic area (MPOA) is a central integrative site for the regulation of male sexual behavior. It receives indirect input from every sensory modality (Simerly and Swanson, 1986) and sends projections to structures critical for the initiation and patterning of copulation (COP) (Simerly and Swanson, 1988). Lesions of the MPOA inhibited male sexual behavior in all studied species; conversely, stimulation facilitated several copulatory measures (for review, see Hull et al., 2002; Hull and Dominguez, 2003). Stimulation of the rats' MPOA also elicited erectile responses (Giuliano et al., 1997) and the urethrogenital reflex, a proposed model for orgasm (Marson and McKenna, 1994).

Furthermore, neural activity in the MPOA increases with sexual activity. Electrophysiological recordings in the MPOA of sexually experienced monkeys showed increased neural activity both when the animal bar-pressed to bring a conspecific female closer and during COP, whereas activity ceased after ejaculation (EJAC) (Oomura et al., 1988). Similar results were observed in rats, with continuously increasing activity during mating, until after EJAC, when activity decreased during the postejaculatory interval (PEI) (Shimura et al., 1994). Similarly, sexual activity increased Fos immunoreactivity (-ir), a measure of cellular activity, in the MPOA of male rats (Baum and Everitt, 1992; Bressler and Baum, 1996; Coolen et al., 1996; for review, see Pfaus and Heeb, 1997), hamsters (Kollack-Walker and Newman, 1997), gerbils (Heeb

Received Sept. 30, 2005; revised Dec. 20, 2005; accepted Dec. 24, 2005.

This work was supported by National Institutes of Health Grants K02-MH01714 and R01-MH40826 to E.M.H. We thank Alison Hood and Anlys Olivera for assisting with microdialysis and behavioral experiments. We also thank Dr. Jessica Brann for comments on this manuscript.

Correspondence should be addressed to Dr. Juan M. Dominguez, Department of Psychology, Florida State University, Tallahassee, FL 32306-1270. E-mail: dominguez@neuro.fsu.edu.

DOI:10.1523/JNEUROSCI.4176-05.2006

Copyright $\odot 2006$ Society for Neuroscience $\quad$ 0270-6474/06/261699-05\$15.00/0 and Yahr, 1996), and musk shrews (Gill et al., 1998). These data support the conclusion that neural activity in the MPOA is important for male sexual behavior.

However, the mechanisms responsible for activation of the MPOA during mating are not known. Because glutamate is the major excitatory neurotransmitter in the CNS, we tested whether glutamate in 2 min microdialysate samples from the MPOA of male rats increases during sexual activity and whether increasing glutamate levels by reverse dialysis of glutamate uptake inhibitors into the MPOA facilitates sexual behavior.

\section{Materials and Methods}

Subjects. Adult male Long-Evans/Blue Spruce rats (Harlan, Indianapolis, IN) were housed individually in large plastic cages. Rats were housed in a climate-controlled room, on a 14/10 h light/dark cycle, with lights off at 11:00 A.M. and on at 9:00 P.M. Food and water were available ad libitum.

Conspecific females were ovariectomized under ketamine hydrochloride $(50 \mathrm{mg} / \mathrm{kg})$ and xylazine hydrochloride $(4 \mathrm{mg} / \mathrm{kg})$ anesthesia. They were brought into behavioral estrus with $10 \mu \mathrm{g}$ of estradiol benzoate $48 \mathrm{~h}$ before and $500 \mu \mathrm{g}$ of progesterone $4 \mathrm{~h}$ before testing. Behavioral receptivity was confirmed by placing the female with a stud male shortly before the test began. Subject males were sexually experienced, having copulated to two EJACs in each of two tests. All procedures were in accordance with the National Institutes of Health Guidelines for the Use of Animals and approved by the Institutional Animal Care and Use Committee of Florida State University.

Stereotaxic surgeries. Sexually experienced male rats $(n=10$ for experiment $1 ; n=16$ for experiment 2) were anesthetized with ketamine hydrochloride $(50 \mathrm{mg} / \mathrm{kg})$ and xylazine hydrochloride $(4 \mathrm{mg} / \mathrm{kg})$ and received guide cannulas aimed at the MPOA. The guide cannulas, made of 23 gauge thin-wall stainless steel tubing, ended $2 \mathrm{~mm}$ above the MPOA [anteroposterior, $2.3 \mathrm{~mm}$; mediolateral, $0.3 \mathrm{~mm}$; dorsoventral, $26.2 \mathrm{~mm}$ (Pellegrino et al., 1979)] and were secured to the skull and skull screws with dental acrylic. Bacitracin (400 U/g) antibiotic was then spread around the wound margins. An obturator, cut the same length as the 
guide cannula, was inserted into the guide cannula until microdialysis experiments began. Aseptic techniques were used throughout all surgical procedures.

Microdialysis and behavior. Animals were allowed a 2 week recovery period after surgery. On the morning of testing, concentric microdialysis probes were implanted through the guide cannulas into the MPOA of subject males. The dialysis membrane (Spectra/Por in vivo microdialysis hollow fibers; Spectrum, Gardena, CA) had an outer diameter of $170 \mu \mathrm{m}$, an inner diameter of $150 \mu \mathrm{m}$, an active dialyzing length of $1 \mathrm{~mm}$, and an 18,000 molecular weight cutoff. A Teflon-covered tether encased the inflow tubing. Artificial CSF (ACSF; in mM: $138 \mathrm{NaCl}, 2.7 \mathrm{KCl}, 0.5$ $\mathrm{MgCl}_{2}, 1.5 \mathrm{KH}_{2} \mathrm{PO}_{4}$, and 1.2 $\mathrm{CaCl}_{2}, \mathrm{pH}$ 6.8; Sigma, St. Louis, MO) was perfused at a rate of $2.0 \mu \mathrm{l} / \mathrm{min}$. Samples were collected every $2 \mathrm{~min}$, immediately frozen $\left(-80^{\circ} \mathrm{C}\right)$, and later assayed using HPLC with electrochemical detection (HPLC-EC). Because the time required for dialysate to progress from the membrane to the collection vial was $\sim 30 \mathrm{~s}$, behaviors that occurred during the last $30 \mathrm{~s}$ of a 2 min interval were recorded as occurring during the following interval.

In experiment 1 , after the probe was implanted, the subject was returned to his home cage, and the probe was attached to the perfusion line. Six hours later, three baseline (BL) samples were collected. A sexually receptive female was then placed in a mesh cage above the male's home cage, where he could see, smell, and hear the female but could not copulate with her [precopulation (PRE) period]. During this period, three dialysis samples were collected. The female was then placed into the male's home cage, where they could copulate; during this period, 20 additional samples were collected (COP period). After the last sample was collected, and if the male was not in a PEI, the female was removed and two final samples were collected (postcopulation period). If the male was in the PEI, the test was extended until he intromitted, after which the female was removed. The following samples were assayed using HPLCEC: BL; PRE; COP, samples that included only mounts and intromissions; EJACs; PEI, collected after the rats ejaculated and before the next intromission; and post-test, samples after the female was removed (POST).

A similar microdialysis procedure was used in experiment 2, except that an experimental group received a mixture of glutamate uptake inhibitors [250 $\mu \mathrm{M}$ Chicago sky blue and $250 \mu \mathrm{M}$ L-trans-2,4-PDC (both from Tocris, Ellisville, MO)] in ACSF by reverse dialysis to increase extracellular glutamate in the MPOA. Control animals received only ACSF. The samples included the following: BL; uptake inhibitor or ACSF alone for control animals (UPTKi); PRE; COP, including mounts and intromissions; EJAC; PEI; and POST.

The following behavioral measures were recorded: mount latency (latency to first mount, or first intromission if not preceded by a mount); intromission latency (latency to the first intromission); EJAC latency (latency from the first intromission to the first EJAC); PEI (interval from EJAC to the ensuing intromission); mount and intromission frequencies (numbers of mounts and intromissions preceding the first EJAC); and EJAC frequency during the test.

After testing, cannula placements were verified histologically. Animals were anesthetized with sodium pentobarbital, and, using the same probe that was used for microdialysis, a dye solution was reverse-dialyzed into the MPOA. Animals were immediately killed with an overdose of sodium pentobarbital, and their brains were removed, frozen, and sectioned (40 $\mu \mathrm{m})$ using a cryostat. Sections including the MPOA were mounted on slides and examined for cannula placement using an Olympus (Tokyo, Japan) microscope.

Chromatography. All microdialysis samples were later analyzed using an LC Packings (San Francisco, CA) chromatographic system, consisting of an Acurate microflow processor and pulse damper, a Valco Instruments (Houston, TX) injector with a $500 \mathrm{nl}$ sample loop, and an Antec microelectrochemical detector, equipped with a microflow cell $(11 \mathrm{nl}$ cell volume), with a glassy carbon working electrode and a $\mathrm{Ag} / \mathrm{AgCl}$ reference electrode. The analytical column was an LC Packings Fusica reversedphase capillary column (300 $\mu \mathrm{m}$ inner diameter, $5 \mathrm{~cm}$ long, packed with $3 \mu \mathrm{m}$ C-18 particles). The working electrode was maintained at an applied potential of $0.7 \mathrm{~V}$ relative to the reference electrode. A Gilson Medical Electronics (Middleton, WI) pump delivered mobile phase $(0.085 \mathrm{M}$
$\mathrm{Na}_{2} \mathrm{HPO}_{4}, 135 \mu \mathrm{M}$ EDTA, and 15\% MeOH, in HPLC-grade water, $\mathrm{pH}$ 6.50 ) through the system at $0.5 \mathrm{ml} / \mathrm{min}$; however, the microflow processor split the flow, so that flow through the analytical column was $\sim 7$ $\mu \mathrm{l} / \mathrm{min}$. Before injection, dialysate underwent precolumn derivatization with ophthaldialdehyde (OPA) to render glutamate electrochemically detectable. Five-microliter of $\beta$-mercaptoethanol were added to an OPA stock solution ( $27 \mathrm{mg}$ of OPA in $1 \mathrm{ml}$ of ethanol diluted with $9 \mathrm{ml}$ of 0.1 $\mathrm{M} \mathrm{Na}_{2} \mathrm{~B}_{4} \mathrm{O}_{7}, \mathrm{pH}$ 9.4). Fresh working solution was obtained by diluting OPA with $\mathrm{Na}_{2} \mathrm{~B}_{4} \mathrm{O}_{7}$ buffer (3:1). One volume of OPA reagent was mixed with two volumes of sample and allowed to react for $2 \mathrm{~min}$ before injecting into the HPLC system. Data were collected using the Gilson Unipoint system controller software (Gilson Medical Electronics), which also controlled the pump parameters.

Data analysis. Samples from each animal were categorized as BL, PRE, COP, EJAC, PEI, or POST and averaged within categories. In experiment 1 , a one-way ANOVA was used to test for significant differences between samples; in experiment 2, a two-way ANOVA was used (treatment by sample), followed by one-way analysis if the two-factor analysis indicated a significant main effect of treatment or interaction. Newman-Keuls tests were used to probe for significant differences among individual means. Also, $t$ tests were used in experiment 2 to probe for differences in behavioral measures between animals receiving uptake inhibitors or ACSF alone. A Pearson's correlation was used to assess the relationship between postejaculatory decline in glutamate and duration of the PEI.

\section{Results}

\section{Sexual activity increases extracellular glutamate in the MPOA} of male rats

Glutamate concentrations in dialysate samples collected during BL measured $185 \pm 14.3 \mathrm{nM}$. In experiment 1 , analysis of percentage of changes from BL revealed a significant effect of sample $\left(F_{(5,170)}=29.934 ; p<0.001\right)$ (Fig. $\left.1 A, B\right)$. Levels of glutamate were significantly higher in COP and EJAC samples compared with $\mathrm{BL}(p<0.05)$ and were highest in EJAC samples compared with all other samples, including $\operatorname{COP}(p<0.001)$. Finally, compared with EJAC samples, levels dropped precipitously during the PEI $(p<0.001)$. Levels returned to BL after mating, when the female was removed.

Analysis with a Pearson's correlation coefficient revealed that the difference in the levels of glutamate between EJAC samples and the first PEI sample correlated with the length of time spent in the refractory period $(r=0.763 ; p<0.0001)$ (Fig. $1 C)$. This suggests that a larger decrease in glutamate after EJAC results in longer time spent in the refractory period, before the animal reinitiates mating.

\section{Dialysis of glutamate uptake inhibitors into the MPOA increases glutamate and facilitates male sexual behavior}

To test directly whether MPOA glutamate facilitates sexual behavior, in experiment 2 we reverse-dialyzed a mixture of glutamate uptake inhibitors while measuring behavior. Analysis of percentage of changes from BL with a two-way ANOVA revealed significant differences attributable to treatment $\left(F_{(1,295)}=\right.$ $188.033 ; p<0.001)$, sample $\left(F_{(6,295)}=29.293 ; p<0.001\right)$, and interaction $\left(F_{(6,295)}=9.525 ; p<0.001\right)$ (Fig. 2). A NewmanKeuls test revealed larger increases in glutamate for animals receiving uptake inhibitors compared with controls. Animals receiving uptake inhibitors had higher glutamate levels compared with BL, in all post-BL samples $\left(F_{(6,156)}=15.12 ; p<0.001\right)$. Animals receiving ACSF alone had significant increases of glutamate in COP and EJAC samples compared with $\mathrm{BL}\left(F_{(6,139)}=\right.$ 31.116; $p<0.001$ ).

Glutamate uptake inhibitors facilitated several measures of sexual behavior (Table 1). Specifically, animals receiving uptake inhibitors displayed significantly more EJACs $\left(t_{(11)}=-4.194\right.$; 


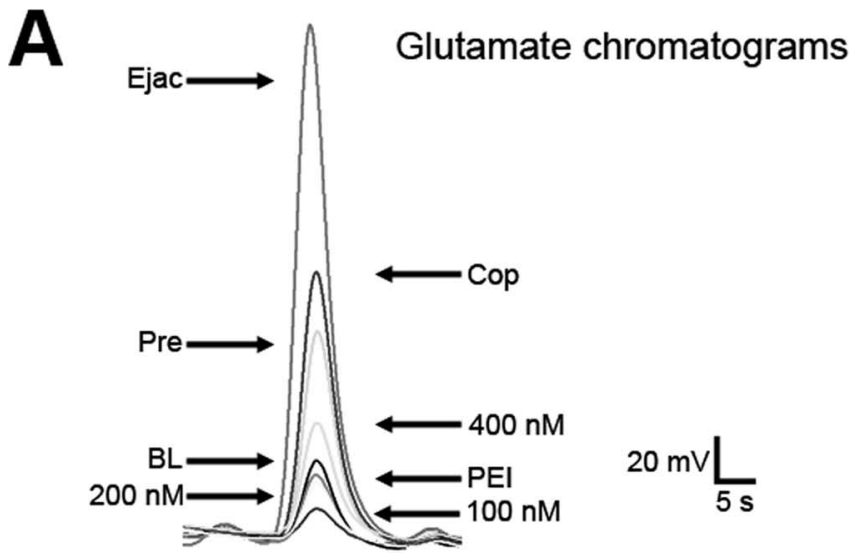

B
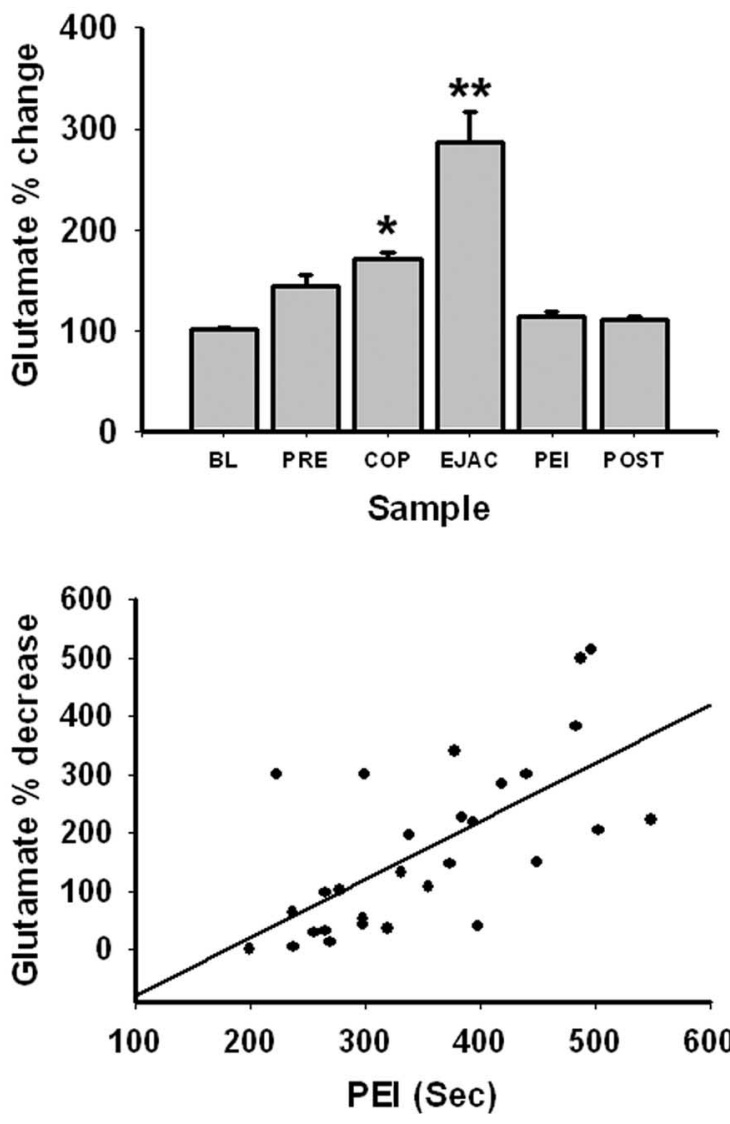

Figure 1. Extracellular MPOA glutamate changes with COP. A, Comparison of chromatograms collected from an animal before and during $C O P$ with standards. $\boldsymbol{B}$. Mean change in MPOA glutamate release during BL, PRE, COP, EJAC, PEI, and POST. Samples were collected at 2 min intervals. Values are expressed as mean \pm SEM. ${ }^{*} p<0.05 ;{ }^{* *} p<0.001$. C, Relationship of postejaculatory decrease in glutamate to duration of PEI $(r=0.763)$.

$p<0.01)$, required less time to reach an EJAC $\left(t_{(11)}=2.507 ; p<\right.$ $0.05)$, and had shorter PEIs $\left(t_{(11)}=2.335 ; p<0.05\right)$ compared with controls.

In experiment 1,8 of 10 animals received probe placements ending in the MPOA; only these samples were included in analyses. Separate analyses of samples collected from animals with missed placements revealed no significant changes of glutamate in response to mating or to the female, suggesting that changes in glutamate were specific to the MPOA. In experiment 2, one animal received cannula placement outside of the MPOA. Also in

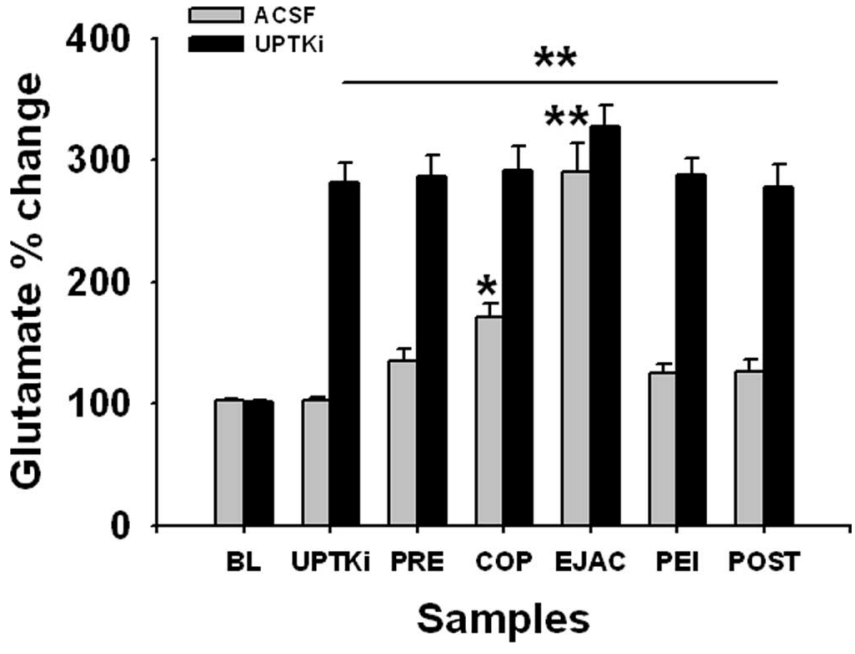

Figure 2. Extracellular MPOA glutamate changes with glutamate uptake inhibitors and COP. Glutamate was increased throughout the period of reverse dialysis of uptake inhibitors compared with $\mathrm{BL}$; there was no additional increase during COP. In control animals, glutamate increased significantly during $\mathrm{COP}$ and during EJAC. Samples were collected at $2 \mathrm{~min}$ intervals. Values are expressed as mean \pm SEM. ${ }^{*} p<0.05 ;{ }^{* *} p<0.001$.

Table 1. Reverse dialysis of glutamate uptake inhibitors facilitated male sexual behavior

\begin{tabular}{lcc}
\hline & ACSF & Glutamate uptake inhibitors \\
\hline Mount frequencies & $2.33 \pm 0.71$ & $3.71 \pm 0.87$ \\
Intromission frequencies & $4.83 \pm 0.60$ & $4.43 \pm 0.57$ \\
EJAC frequencies & $2.83 \pm 0.31$ & $4.29 \pm 0.18^{* *}$ \\
Mount latencies (s) & $16.33 \pm 1.89$ & $12.71 \pm 3.34$ \\
Intromission latencies (s) & $29.17 \pm 8.75$ & $24.86 \pm 8.10$ \\
EJAC latencies (s) & $309.83 \pm 57.32$ & $158.71 \pm 26.99^{*}$ \\
PEI (s) & $435.83 \pm 60.45$ & $298.57 \pm 17.75^{*}$ \\
\hline
\end{tabular}

Animals receiving uptake inhibitors displayed more EJACs, required less time to reach an $E J A C$, and required less time to reinitiate mating after ejaculating. Values are expressed as mean $\pm \mathrm{SEM}$. ${ }^{*} p<0.05 ;{ }^{* *} p<0.01$.

this experiment, two animals chewed through their microdialysis tubing during testing; this resulted in inadequate dialysate volume for analysis, and their samples were also excluded. After exclusion of animals with missed placements or interrupted microdialysis flow, 8 animals remained in experiment 1 and 13 animals remained in experiment $2(n=6$ for controls and $n=7$ for the uptake inhibitor group).

\section{Discussion}

In experiment 1 , sexual activity increased levels of glutamate in the MPOA of male rats. This increase was highest ( $~ 300 \%$ of BL) in samples collected when the animals ejaculated; levels then dropped precipitously in samples collected during the PEI. This drop in glutamate between EJAC and subsequent PEI samples was highly correlated with time spent in the PEI, indicating that a larger decrease in glutamate after EJAC is associated with a longer PEI. These data suggest that glutamate in the MPOA facilitates male sexual behavior and that the extent of glutamate decrease in the MPOA after EJAC regulates the duration of the PEI.

This conclusion was further supported by results of experiment 2 , in which reverse dialysis of glutamate uptake inhibitors into the MPOA increased levels of glutamate and facilitated sexual behavior. Specifically, animals with enhanced extracellular glutamate displayed more EJACs, required less time to reach EJAC, and required less time to reinitiate sexual activity after ejaculating. 
It is well established that activity in the MPOA facilitates male sexual behavior. Sexual behavior is severely compromised after removal of the MPOA. Fewer than $30 \%$ of rats with MPOA lesions mounted, fewer than $15 \%$ intromitted, and fewer than $3 \%$ ejaculated, whereas $100 \%$ of control animals displayed all three behaviors (Liu et al., 1997). Conversely, stimulation of the MPOA enhances sexual activity. In one study, animals receiving MPOA stimulation were more likely to achieve three and four EJACs in a 30 min test compared with animals not receiving stimulation (Rodriguez-Manzo et al., 2000). Similarly, mating increased Fos-ir in the MPOA of males (for review, see Hull et al., 2002). In one study, animals that mounted and intromitted had more Fos-ir than animals that did not; those that ejaculated had more Fos than those that only mounted and intromitted (Coolen et al., 1996).

Electrophysiological recordings also indicate that MPOA activity is important for male sexual behavior. In macaque monkeys, neural activity increased in the presence of a female, increased further with erections and intromission, and continued to increase until the male reached an EJAC; after EJAC, activity decreased to the lowest level and remained low for $\sim 10 \mathrm{~min}$ before it began to rise again (Oomura et al., 1988). Similar recordings in the MPOA of male rats indicate a progressive increase in the number of cells activated while mating; after EJAC, activity decreased significantly (Shimura et al., 1994).

Our results suggest that glutamate in the MPOA is at least partly responsible for driving neural activity in the MPOA. In experiment 1 , glutamate activity increased during mating and peaked with EJAC; in experiment 2, enhanced glutamatergic activity in the MPOA facilitated sexual behavior. In addition, the precipitous decrease in glutamate levels between EJAC and the PEI may regulate time spent in the PEI.

It has long been suspected that increased MPOA glutamate facilitates male sexual behavior. Administration of glutamate directly into the MPOA of anesthetized rats increased erectile responses (Giuliano et al., 1997) and the urethrogenital reflex, proposed as a model of orgasm (Marson and McKenna, 1994). Also, MPOA microinjections of dizocilpine (MK-801), an antagonist at NMDA glutamate receptors, inhibited $50 \mathrm{kHz}$ ultrasonic vocalizations that were evoked in anticipation of a receptive female; therefore, glutamate in the MPOA might also play a role in regulating appetitive aspects of mating (Brudzynski and Pniak, 2002).

After EJAC, males undergo a period of sexual quiescence, during which the male does not approach the female and displays little behavior. Because males are unresponsive to sexually exciting stimuli during the initial portion of the PEI, it has been suggested that this constitutes an absolute refractory period, whereas the later phase of the PEI constitutes a relative refractory period, during which stimuli such as tail pinch, mild shock, and robust solicitation by a novel female can result in further COP (for review, see Hull et al., 2002). The strong correlation between time spent in the PEI and decreases in glutamate levels after EJAC reported here strongly suggest that mating-induced glutamate activity in the MPOA mediates, at least in part, sexual quiescence associated with the PEI. Specifically, we propose that a larger decrease in glutamate after EJAC lengthens the PEI, making it difficult for sexually exciting stimuli to evoke a response. It is not clear what input induces the precipitous decline in MPOA glutamate after EJAC. However, EJAC-related serotonin release in the lateral hypothalamus inhibits dopamine (DA) release in the nucleus accumbens (Lorrain et al., 1999). Perhaps similar mechanisms influence glutamate release in the MPOA.
It is interesting that, although reverse dialysis of glutamate uptake inhibitors increased glutamate levels as much as did EJAC, animals receiving those inhibitors did not ejaculate immediately. One explanation may be that the volume of MPOA tissue directly affected by microdialysis is small. Thus, a more widespread activation may be necessary to trigger EJAC. In addition, control of EJAC may require permissive input from other brain areas, including the medial amygdala (MeA) (for review, see Hull et al., 2002).

The mechanisms in the MPOA altered by glutamate are not entirely understood. However, stimulation of NMDA receptors appears to be important. Nearly $100 \%$ of cells containing matinginduced Fos-ir also contained NMDA receptors; in addition, mating increased phosphorylation of NMDA receptors, suggesting that NMDA receptors were indeed activated by mating (Dominguez et al., 2003). Moreover, microinjections of the NMDA antagonist MK-801 into the MPOA impaired EJACs (Dominguez et al., 2003; Vigdorchik et al., 2003) and reduced the number of cells containing mating-induced Fos-ir (Dominguez and Coolen, 2004). In addition to NMDA receptor-mediated effects, other glutamate receptors, including both ionotropic and metabotropic receptors, may contribute to the control of mating by the MPOA.

In addition to its direct excitatory role, glutamate might also regulate sexual behavior by mediating the release of other transmitters. For example, numerous studies report that DA in the MPOA is an important facilitator of male sexual behavior (for review, see Dominguez and Hull, 2005). Specifically, microinjections of DA agonists into the MPOA facilitated sexual behavior, whereas antagonists impaired it. Moreover, DA in the MPOA increased in response to sexually exciting stimuli and during mating (Hull et al., 1995; Dominguez et al., 2001). Glutamate may play a role in regulating this DA release. Reverse dialysis of glutamate into the MPOA increased extracellular DA; this increase was prevented by coadministration of a nitric oxide synthase antagonist (Dominguez et al., 2004). These results are relevant to the present findings because glutamate, acting via NMDA receptors, opens $\mathrm{Ca}^{2+}$ channels; this $\mathrm{Ca}^{2+}$ influx can then activate calcium calmodulin, which in turn activates nitric oxide synthase. Therefore, activation of NMDA receptors by glutamate in the MPOA increases levels of extracellular DA in the MPOA by increasing nitric oxide production; increased DA release, in turn, enhances male sexual behavior (for review, see Dominguez and Hull, 2005). In addition to effects of glutamate on DA activity, it is also possible that DA influences glutamatergic function. Experiments are planned to investigate the interactions among these and other transmitters.

Because availability of techniques to visualize glutamate has been limited, the sources of glutamate to the MPOA are not entirely clear. However, a recent study that used injections of $\left[{ }^{3} \mathrm{H}\right] \mathrm{D}$-aspartate to map putative sources of glutamate to the MPOA indicated that possible sources of glutamate include the lateral septum, the bed nucleus of the stria terminalis (BNST), the MeA, the MPOA itself, and paraventricular, suprachiasmatic, ventromedial, arcuate, ventral premammillary, supramammilary, and thalamic paraventricular nuclei (Kocsis et al., 2003). Sources of glutamatergic input to the MPOA include brain regions that are activated by sexually relevant stimuli and are also sensitive to hormonal stimulation, including the MeA and BNST. These results are consistent with observations that some anterogradely labeled axons from the MeA, and many from the BNST, were immunoreactive for the vesicular glutamate transporter, an indicator of glutamatergic terminals (Dominguez et al., 2004), 
suggesting that the MeA and BNST have at least some glutamatergic afferents to the MPOA.

Testosterone reduces the refractory period of neurons in the MeA-stria terminalis continuum that project to the MPOA of male rats (Kendrick and Drewett, 1979), suggesting that circulating testosterone increases the firing rate in these cells. If the MeAstria terminalis continuum is a source of glutamate to the MPOA, as indicated by tract-tracing studies, then one mechanism by which testosterone enhances sexual behavior may be by decreasing refractory periods of glutamatergic neurons in the MeABNST, thereby increasing glutamatergic stimulation in the MPOA, which enhances sexual behavior, as shown here, by facilitating EJAC and decreasing the postejaculatory refractory period.

In summary, increased neural activity in the MPOA facilitates male sexual behavior. We report that glutamate is at least partly responsible for this mating-induced activation. Furthermore, glutamate in the MPOA might be particularly important for EJAC, as evidenced by the strong increase in activity during EJAC (in experiment 1) and facilitated ejaculatory response to enhanced glutamate activity (in experiment 2). In addition, these data suggest that decreased glutamate in the MPOA after EJAC might play a role in regulating the PEI.

\section{References}

Baum MJ, Everitt BJ (1992) Increased expression of c-fos in the medial preoptic area after mating in male rats: role of afferent inputs from the medial amygdala and midbrain central tegmental field. Neuroscience 50:627-646.

Bressler SC, Baum MJ (1996) Sex comparison of neuronal Fos immunoreactivity in the rat vomeronasal projection circuit after chemosensory stimulation. Neuroscience 71:1063-1072.

Brudzynski SM, Pniak A (2002) Social contacts and production of $50-\mathrm{kHz}$ short ultrasonic calls in adult rats. J Comp Psychol 116:73-82.

Coolen LM, Peters HJ, Veening JG (1996) Fos immunoreactivity in the rat brain following consummatory elements of sexual behavior: a sex comparison. Brain Res 738:67-82.

Dominguez J, Riolo JV, Xu Z, Hull EM (2001) Regulation by the medial amygdala of copulation and medial preoptic dopamine release. J Neurosci 21:349-355.

Dominguez JM, Coolen LM (2004) Activation of NMDA receptors in the medial preoptic nucleus is essential for the expression of Fos and male sexual behavior. Horm Behav 46:129.

Dominguez JM, Hull EM (2005) Dopamine, the medial preoptic area, and male sexual behavior. Physiol Behav 86:356-368.

Dominguez JM, Balfour ME, Coolen LM (2003) Copulation-induced activation of NMDA receptor containing neurons in the medial preoptic nucleus. Horm Behav 44:46.

Dominguez JM, Muschamp JW, Schmich JM, Hull EM (2004) Nitric oxide mediates glutamate-evoked dopamine release in the medial preoptic area. Neuroscience 125:203-210.
Gill CJ, Wersinger SR, Veney SL, Rissman EF (1998) Induction of Fos-like immunoreactivity in musk shrews after mating. Brain Res 811:21-28.

Giuliano F, Bernabe J, Brown K, Droupy S, Benoit G, Rampin O (1997) Erectile response to hypothalamic stimulation in rats: role of peripheral nerves. Am J Physiol 273:R1990-R1997.

Heeb MM, Yahr P (1996) c-Fos immunoreactivity in the sexually dimorphic area of the hypothalamus and related brain regions of male gerbils after exposure to sex-related stimuli or performance of specific sexual behaviors. Neuroscience 72:1049-1071.

Hull EM, Dominguez JM (2003) Sex behavior. In: Handbook of psychology, biological psychology, Vol 3 (Gallagher M, Nelson RJ, Weiner IB, eds), pp 321-354. Hoboken, NJ: Wiley.

Hull EM, Du J, Lorrain DS, Matuszewich L (1995) Extracellular dopamine in the medial preoptic area: implications for sexual motivation and hormonal control of copulation. J Neurosci 15:7465-7471.

Hull EM, Meisel RL, Sachs BD (2002) Male sexual behavior. In: Hormones, brain and behavior (Pfaff DW, Arnold AP, Etgen AM, Fahrbach SE, Rubin RT, eds), pp 3-137. San Diego: Academic.

Kendrick KM, Drewett RF (1979) Testosterone reduces refractory period of stria terminalis neurons in the rat brain. Science 204:877-879.

Kocsis K, Kiss J, Csaki A, Halasz B (2003) Location of putative glutamatergic neurons projecting to the medial preoptic area of the rat hypothalamus. Brain Res Bull 61:459-468.

Kollack-Walker S, Newman SW (1997) Mating-induced expression of c-fos in the male Syrian hamster brain: role of experience, pheromones, and ejaculations. J Neurobiol 32:481-501.

Liu YC, Salamone JD, Sachs BD (1997) Lesions in medial preoptic area and bed nucleus of stria terminalis: differential effects on copulatory behavior and noncontact erection in male rats. J Neurosci 17:5245-5253.

Lorrain DS, Riolo JV, Matuszewich L, Hull EM (1999) Lateral hypothalamic serotonin inhibits nucleus accumbens dopamine: implications for sexual satiety. J Neurosci 19:7648-7652.

Marson L, McKenna KE (1994) Stimulation of the hypothalamus initiates the urethrogenital reflex in male rats. Brain Res 638:103-108.

Oomura Y, Aou S, Koyama Y, Fujita I, Yoshimatsu H (1988) Central control of sexual behavior. Brain Res Bull 20:863-870.

Pellegrino LJ, Pellegrino AS, Cushman AJ (1979) A stereotaxic atlas of the rat brain. New York: Plenum.

Pfaus JG, Heeb MM (1997) Implications of immediate-early gene induction in the brain following sexual stimulation of female and male rodents. Brain Res Bull 44:397-407.

Rodriguez-Manzo G, Pellicer F, Larsson K, Fernandez-Guasti A (2000) Stimulation of the medical preoptic area facilitates sexual behavior but does not reverse sexual satiation. Behav Neurosci 114:553-560.

Shimura T, Yamamoto T, Shimokochi M (1994) The medial preoptic area is involved in both sexual arousal and performance in male rats: reevaluation of neuron activity in freely moving animals. Brain Res 640:215-222.

Simerly RB, Swanson LW (1986) The organization of neural inputs to the medial preoptic nucleus of the rat. J Comp Neurol 246:312-342.

Simerly RB, Swanson LW (1988) Projections of the medial preoptic nucleus: a Phaseolus vulgaris leucoagglutinin anterograde tract-tracing study in the rat. J Comp Neurol 270:209-242.

Vigdorchik A, Lagoda G, Hull EM (2003) A glutamate receptor antagonist microinjected into the MPOA impairs male sexual behavior. Horm Behav 44:82. 